\title{
Elastic Constants for Various Classes of Solids at High Temperature
}

\author{
Q. $\operatorname{LIU}^{a, *}$ AND Q. $\mathrm{HE}^{b}$ \\ ${ }^{a}$ Department of Physics, Anhui University, Hefei 230039, China \\ ${ }^{b}$ Shanghai Institute of Technical Physics, Chinese Academy of Science \\ Shanghai 200083, China
}

(Received May 15, 2007)

\begin{abstract}
In the present communication, a new relationship for the temperature dependences of elastic constants are developed using a new expression for the temperature dependence of bulk modulus and the formulation derived from Tallon's model. The proposed relationship is applied to study elastic constants of $\mathrm{NaCl}, \mathrm{KCl}, \mathrm{CaF}_{2}, \mathrm{MgO}, \mathrm{CaO}$, and $\mathrm{Mg}_{2} \mathrm{SiO}_{4}$. The result obtained for second-order elastic constants are found to compare well with the experimental data.
\end{abstract}

PACS numbers: 62.20.Dc, 91.60.Ki

\section{Introduction}

The knowledge of elastic constants is essential to interpret the thermodynamic and elastic dimensions of solids at high temperatures. The elasticity offers more information than the volume in interpreting the temperature dependence of equation of state because the compressibility is defined by the derivative of volume. The elastic constants also provide a ground for examining of Earth's deep interior [1]. The behaviors of elastic constants under the influence of high temperature have attracted the attention of experimental [2-4] as well as theoretical workers [5-8]. It has been observed that the methods based on the theory of interionic potentials, either two-body or three-body, are very tedious [5-8] and involve a lot of computational work in addition to various approximations. Thus, it may be useful to propose a simple and straightforward method for predicting high-temperature elastic constants, which is the purpose of the present paper.

In this paper, first we develop a new expression for the temperature dependence of bulk modulus by using fundamental thermodynamic relations simultaneously assuming that the volume thermal expansion coefficient is a quadratic

*corresponding author; e-mail: quanliu@ah172.com.cn 
function of temperature. By combining the new expression with the formulation derived from Tallon's model [9], then we calculate the temperature dependences of elastic constants for $\mathrm{NaCl}, \mathrm{KCl}, \mathrm{CaF}_{2}, \mathrm{MgO}, \mathrm{CaO}$, and $\mathrm{Mg}_{2} \mathrm{SiO}_{4}$. The results are found to yield a close agreement with the available experimental data, which in turn, reveals the validity of the present work.

\section{A new expression for the temperature dependence of bulk modulus}

The Anderson-Gruneisen parameter, which plays an important role in understanding the anharmonic properties of ionic solids, is defined as follows [10]:

$$
\delta_{T}=-\frac{1}{\alpha B_{T}}\left(\frac{\partial B_{T}}{\partial T}\right)_{P},
$$

where $\alpha$ and $B_{T}$ are the coefficient of volume thermal expansion and isothermal bulk modulus, respectively.

They are defined as

$$
\alpha=\frac{1}{V}\left(\frac{\partial V}{\partial T}\right)_{P}
$$

and

$$
B_{T}=\frac{1}{V}\left(\frac{\partial P}{\partial V}\right)_{T} .
$$

A method to estimate the temperature dependence of the bulk modulus has been developed with the approximation that $\alpha$ depends linearly on temperature [11]. But we find that, in the case of $\mathrm{NaCl}$ whose experimental results had been reported, the values of $B_{T}$ calculated in Ref. [11] is adequate for small expansions. The percentage differences increase with increasing temperature and become $9.75 \%$ at melting point. Thus, the approximation that $\alpha$ depends linearly on temperature is valid only for small expansions that are in the low temperature range.

Through comparing and analyzing some experimental data, this paper puts forward that $\alpha$ depends quadratically on temperature, which can be written as follows:

$$
\alpha=\alpha_{0}+\alpha_{0}^{\prime}\left(T-T_{0}\right)+\frac{\alpha_{0}^{\prime \prime}}{2}\left(T-T_{0}\right)^{2},
$$

where $\alpha_{0}$ is the value of $\alpha$ at $T_{0}$. $\alpha_{0}^{\prime}$ and $\alpha_{0}^{\prime \prime}$ are, respectively, the first- and second-order temperature derivatives of $\alpha$ at initial temperature $T=T_{0}=300 \mathrm{~K}$. If one assumes that the product $\alpha B_{T}$ remains constant [12], then we get from Eq. (1) the following approximate formulation:

$$
\delta_{T}=\frac{1}{\alpha^{2}}\left(\frac{\partial \alpha}{\partial T}\right)_{P} .
$$

Assuming $\delta_{T}$ to be independent of $T$ [12], we can get the following relations:

$$
\alpha_{0}^{\prime}=\left(\frac{\partial \alpha}{\partial T}\right)_{P}=\delta_{T} \alpha_{0}^{2}
$$

and 


$$
\alpha_{0}^{\prime \prime}=\left(\frac{\partial^{2} \alpha}{\partial T^{2}}\right)_{P}=2 \delta_{T_{0}}^{2} \alpha_{0}^{3}
$$

where $\delta_{T_{0}}$ is the value of $\delta_{T}$ at $T_{0}$ and at atmospheric pressure, i.e. at $P=0$. Therefore Eq. (4) can be rewritten as follows:

$$
\alpha=\alpha_{0}+\delta_{T_{0}} \alpha_{0}^{2}\left(T-T_{0}\right)+\delta_{T_{0}}^{2} \alpha_{0}^{3}\left(T-T_{0}\right)^{2} .
$$

Substituting the value of $\alpha$ from Eq. (8) in Eq. (1), we get

$$
\frac{\mathrm{d} B_{T}}{B_{T}}=-\delta_{T_{0}}\left[\alpha_{0}+\delta_{T_{0}} \alpha_{0}^{2}\left(T-T_{0}\right)+\delta_{T_{0}}^{2} \alpha_{0}^{3}\left(T-T_{0}\right)^{2}\right] \mathrm{d} T .
$$

Integrating Eq. (9),we finally get the following expression for $B_{T}(T)$ :

$\frac{B_{T}}{B_{T_{0}}}=\exp \left(-\delta_{T_{0}} \alpha_{0}\left(T-T_{0}\right)\left(1+\frac{\delta_{T_{0}} \alpha_{0}\left(T-T_{0}\right)}{2}+\frac{\delta_{T_{0}}^{2} \alpha_{0}^{2}\left(T-T_{0}\right)^{2}}{3}\right)\right)$,

where $B_{T_{0}}$ is the value of $B_{T}$ at initial temperature $T=T_{0}=300 \mathrm{~K}$ and at atmospheric pressure.

\section{The expressions for the temperature dependence of elastic constants}

Grover et al. [13] used a non-standard definition of $\delta_{T}$ and recalled it the parameter $g$ as given below

$$
g=-\frac{V_{0}}{B_{T}}\left(\frac{\partial B_{T}}{\partial V}\right)_{P}
$$

where $V_{0}$ is the value of $V$ at $P=0$. Equation (11) when generalized reads as follows:

$$
g_{m}=-\frac{V_{0}}{M}\left(\frac{\partial M}{\partial V}\right)_{P}
$$

where $M$ represents any of the elastic moduli, such as $C_{11}, C_{12}, C_{44},\left(C_{11}-C_{12}\right) / 2$ or $B_{T}$. Tallon [9] has expressed Eq. (12) as follows:

$$
\frac{M}{M_{0}}=\exp \left(-g_{m}\left(\frac{V}{V_{0}}-1\right)\right)
$$

Equation (13) may be used to determine the temperature dependence of elastic constants. Following the method of generalization as used by Tallon [9] to get Eq. (13), we can generalize Eq. (10) as follows:

$$
\frac{M}{M_{0}}=\exp \left(-\delta_{M_{0}} \alpha_{0}\left(T-T_{0}\right)\left(1+\frac{\delta_{M_{0}} \alpha_{0}\left(T-T_{0}\right)}{2}+\frac{\delta_{M_{0}}^{2} \alpha_{0}^{2}\left(T-T_{0}\right)^{2}}{3}\right)\right) .
$$

In Eq. (14), $\delta_{M_{0}}$ should vary according to the elastic moduli selected. On the basis of Eq. (14), the relevant expression for elastic constants may be written collectively as follows:

$$
\frac{C_{i j}}{C_{i j_{0}}}=\exp \left(-\delta_{i j_{0}} \alpha_{0}\left(T-T_{0}\right)\left(1+\frac{\delta_{i j_{0}} \alpha_{0}\left(T-T_{0}\right)}{2}+\frac{\delta_{i j_{0}}^{2} \alpha_{0}^{2}\left(T-T_{0}\right)^{2}}{3}\right)\right) .
$$

Here $\delta_{i j_{0}}$ is given by Eq. (1) as defined by Kumar and Bedi [14]. 


\section{Results and discussions}

Equation (10), obtained by the authors, is used to compute the values of $B_{T}$ at different $T$, using the input data $[2,5,15]$ given in Table I. The results obtained from Ref. [11] are also included in Table II for the sake of comparison. It is found that Eq. (10) improves the results as compared with Ref. [11]. Thus, Eq. (10) proposed by the authors seems to be suitable for the temperature dependence of bulk modulus. This encouraged the authors to extend Eq. (10) for the study of the variation of elastic moduli. Equation (10) may be generalized in the form of Eq. (14). Equation (14) may be written for the second-order elastic constants (SOEC) in

TABLE I

Values of input parameters $[2,5,15] . \quad \alpha_{0}$ in $\left[10^{-6} \mathrm{~K}^{-1}\right]$.

\begin{tabular}{c|c|c|c|c|c|c}
\hline \hline & $\mathrm{NaCl}$ & $\mathrm{KCl}$ & $\mathrm{CaF}_{2}$ & $\mathrm{MgO}$ & $\mathrm{CaO}$ & $\mathrm{MgSiO}_{4}$ \\
\hline$\alpha_{0}$ & 118 & 110 & 56 & 31.2 & 30.4 & 27.2 \\
$\delta_{11_{0}}$ & 6.27 & 7.48 & 3.43 & 6.27 & 7.3 & 4.06 \\
$\delta_{12_{0}}$ & 0.051 & -3.31 & 4.61 & -2.49 & -2.0 & 6.00 \\
$\delta_{44_{0}}$ & 2.34 & 3.05 & 6.50 & 2.57 & 2.9 & 7.50 \\
$\delta_{22_{0}}$ & - & - & - & - & - & 5.07 \\
$\delta_{23_{0}}$ & - & - & - & - & - & 3.20 \\
$\delta_{31_{0}}$ & - & - & - & - & - & 5.10 \\
$\delta_{33_{0}}$ & - & - & - & - & - & 4.50 \\
$\delta_{55_{0}}$ & -- & - & - & - & - & 6.60 \\
$\delta_{66_{0}}$ & - & - & - & - & - & 7.50
\end{tabular}

TABLE II

Values of bulk modulus $B_{T}$ (in kbar) at different temperatures $T$ for $\mathrm{NaCl}$. $\delta_{T_{0}}=5.95$ [11].

\begin{tabular}{c|c|c|c}
\hline \hline \multirow{2}{*}{$\mathrm{T}[\mathrm{K}]$} & \multicolumn{3}{|c}{$B_{T}$} \\
\cline { 2 - 4 } & Ref. [11] & This work & Exp. [2] \\
\hline 300 & 240.0 & 240.0 & 240.0 \\
400 & 219.7 & 223.2 & 224.1 \\
500 & 199.9 & 206.3 & 205.0 \\
600 & 18.09 & 189.6 & 188.0 \\
700 & 151.2 & 172.9 & 174.0 \\
800 & 145.6 & 156.6 & 156.0 \\
900 & 128.8 & 140.6 & 140.9 \\
$T_{m}(1050 \mathrm{~K})$ & 107.4 & 117.5 & 119.0 \\
\hline Percentage & & & \\
deviation at $T_{m}$ & 9.75 & 1.26 & -
\end{tabular}


TABLE III

NaCl: Calculated values of $C_{11}, C_{12}$, and $C_{44}$ (in GPa) at different temperatures along with the experimental data [2] obtained under adiabatic condition.

\begin{tabular}{c|c|c|c|c|c|c}
\hline \hline \multirow{2}{*}{$T[\mathrm{~K}]$} & \multicolumn{2}{|c|}{$C_{11}$} & \multicolumn{2}{c|}{$C_{12}$} & \multicolumn{2}{c}{$C_{44}$} \\
\cline { 2 - 7 } & Eq. (15) & {$[2]$} & Eq. (15) & {$[2]$} & Eq. (15) & {$[2]$} \\
\hline 300 & 49.5 & 49.5 & 13.2 & 13.2 & 12.79 & 12.79 \\
350 & 47.7 & 47.6 & 13.20 & 13.3 & 12.61 & 12.62 \\
400 & 45.8 & 45.8 & 13.19 & 13.4 & 12.44 & 12.43 \\
450 & 44.0 & 44.1 & 13.19 & 13.5 & 12.26 & 12.26 \\
500 & 42.2 & 42.4 & 13.18 & 13.6 & 12.08 & 12.09 \\
550 & 40.4 & 40.5 & 13.18 & 13.5 & 11.91 & 11.90 \\
600 & 38.5 & 38.7 & 13.18 & 13.2 & 11.73 & 11.71 \\
650 & 36.7 & 37.0 & 13.17 & 13.1 & 11.55 & 11.52 \\
700 & 34.9 & 35.4 & 13.17 & 13.1 & 11.38 & 11.31 \\
750 & 33.2 & 33.7 & 13.16 & 12.9 & 11.20 & 11.10
\end{tabular}

TABLE IV

$\mathrm{KCl}$ : Calculated values of $C_{11}, C_{12}$, and $C_{44}$ (in $\mathrm{GPa}$ ) at different temperatures along with the experimental data [2] obtained under adiabatic condition.

\begin{tabular}{c|c|c|c|c|c|c}
\hline \hline \multirow{2}{*}{$T[\mathrm{~K}]$} & \multicolumn{2}{|c|}{$C_{11}$} & \multicolumn{2}{c|}{$C_{12}$} & \multicolumn{2}{c}{$C_{44}$} \\
\cline { 2 - 7 } & Eq. (15) & {$[2]$} & Eq. (15) & {$[2]$} & Eq. (15) & {$[2]$} \\
\hline 300 & 40.1 & 40.1 & 6.6 & 6.6 & 6.35 & 6.35 \\
350 & 38.5 & 38.4 & 6.7 & 6.8 & 6.24 & 6.28 \\
400 & 36.8 & 36.9 & 6.8 & 7.0 & 6.14 & 6.21 \\
450 & 35.2 & 35.4 & 7.0 & 7.1 & 6.03 & 6.15 \\
500 & 33.5 & 33.8 & 7.1 & 7.2 & 5.92 & 6.11 \\
550 & 31.9 & 32.3 & 7.2 & 7.3 & 5.82 & 6.05 \\
600 & 30.2 & 31.1 & 7.3 & 7.5 & 5.71 & 5.96 \\
650 & 28.6 & 29.7 & 7.4 & 7.7 & 5.60 & 5.87 \\
700 & 27.0 & 28.2 & 7.6 & 7.7 & 5.50 & 5.79 \\
750 & 25.4 & 26.6 & 7.7 & 7.7 & 5.39 & 5.69 \\
800 & 23.9 & 25.2 & 7.8 & 7.8 & 5.29 & 5.57 \\
850 & 22.3 & 23.5 & 7.9 & 7.7 & 5.18 & 5.57
\end{tabular}

the form of Eq. (15) which is used to compute the temperature dependence of SOEC of NaCl, $\mathrm{KCl}, \mathrm{CaF}_{2}, \mathrm{MgO}, \mathrm{CaO}$, and $\mathrm{Mg}_{2} \mathrm{SiO}_{4}$. The results thus obtained are given in Tables III-VIII along with the experimental data $([2,5,15]$ and $[16])$. 
TABLE V

$\mathrm{CaF}_{2}$ : Calculated values of $C_{11}, C_{12}$, and $C_{44}$ (in $\mathrm{GPa}$ ) at different temperatures along with the experimental data [16] obtained under adiabatic condition.

\begin{tabular}{c|c|c|c|c|c|c}
\hline \hline$T[\mathrm{~K}]$ & \multicolumn{2}{|c|}{$C_{11}$} & \multicolumn{2}{c|}{$C_{12}$} & \multicolumn{2}{c}{$C_{44}$} \\
\cline { 2 - 7 } & Eq. (15) & {$[16]$} & Eq. (15) & {$[16]$} & Eq. (15) & {$[16]$} \\
\hline 300 & 165.5 & 165.5 & 40.7 & 40.7 & 33.5 & 33.5 \\
400 & 162.3 & 162.6 & 39.6 & 39.3 & 32.3 & 32.6 \\
500 & 159.1 & 160.6 & 38.6 & 38.6 & 31.1 & 32.0 \\
600 & 156.0 & 158.0 & 37.5 & 38.2 & 29.8 & 31.3 \\
700 & 152.8 & 154.6 & 36.5 & 37.5 & 28.6 & 30.6 \\
800 & 149.6 & 150.0 & 35.4 & 36.8 & 27.4 & 30.0 \\
900 & 146.4 & 146.6 & 34.4 & 35.7 & 26.2 & 28.6 \\
1000 & 143.3 & 142.0 & 33.4 & 34.3 & 25.0 & 27.0 \\
1100 & 140.1 & 138.0 & 32.3 & 32.9 & 23.8 & 26.2 \\
1200 & 136.9 & 132.0 & 31.3 & 32.2 & 22.6 & 25.3
\end{tabular}

TABLE VI

$\mathrm{MgO}$ : Calculated values of $C_{11}, C_{12}$, and $C_{44}$ (in GPa) at different temperatures along with the experimental data [2] obtained under adiabatic condition.

\begin{tabular}{c|c|c|c|c|c|c}
\hline \hline \multirow{2}{*}{$T[\mathrm{~K}]$} & \multicolumn{2}{|c|}{$C_{11}$} & \multicolumn{2}{c|}{$C_{12}$} & \multicolumn{2}{c}{$C_{44}$} \\
\cline { 2 - 7 } & Eq. (15) & {$[2]$} & Eq. (15) & {$[2]$} & Eq. (15) & {$[2]$} \\
\hline 300 & 298.96 & 298.96 & 96.42 & 96.42 & 157.13 & 157.13 \\
400 & 293.11 & 292.94 & 97.17 & 97.17 & 155.87 & 155.78 \\
600 & 281.41 & 280.62 & 98.67 & 98.00 & 153.35 & 152.84 \\
800 & 269.72 & 268.22 & 100.17 & 98.54 & 150.83 & 149.68 \\
1000 & 258.05 & 255.74 & 101.66 & 98.74 & 148.31 & 146.52 \\
1200 & 246.39 & 243.32 & 103.16 & 98.38 & 145.79 & 143.06 \\
1400 & 234.78 & 230.96 & 104.16 & 97.56 & 143.27 & 139.54 \\
1600 & 223.22 & 219.04 & 106.16 & 96.44 & 140.76 & 136.24 \\
1800 & 211.75 & 208.18 & 107.66 & 95.02 & 138.24 & 133.12
\end{tabular}

In the case of $\mathrm{NaCl}, \mathrm{KCl}, \mathrm{CaF}_{2}, \mathrm{MgO}$, and $\mathrm{CaO}$, there are only three SOEC viz. $C_{11}, C_{12}, C_{44}$. The results obtained for the elastic constants for these five solids under study present reasonably good agreement with the available experimental data $\left([2,5]\right.$ and [15]). And from the above, the variation of $C_{11}$ with temperature is found to be larger as compared with $C_{12}$ and $C_{44}$. The constant $C_{11}$ represents elasticity in length. A longitudinal strain produces a change in volume without a change in shape. The volume change is highly related to the 
TABLE VII

$\mathrm{CaO}$ : Calculated values of $C_{11}, C_{12}$, and $C_{44}$ (in GPa) at different temperatures along with the experimental data [2] obtained under adiabatic condition.

\begin{tabular}{c|c|c|c|c|c|c}
\hline \hline$T[\mathrm{~K}]$ & \multicolumn{2}{|c|}{$C_{11}$} & \multicolumn{2}{c|}{$C_{12}$} & \multicolumn{2}{c}{$C_{44}$} \\
\cline { 2 - 7 } & Eq. (15) & {$[2]$} & Eq. (15) & {$[2]$} & Eq. (15) & {$[2]$} \\
\hline 300 & 220.5 & 220.5 & 57.67 & 57.67 & 80.03 & 80.03 \\
400 & 215.6 & 215.7 & 58.02 & 57.96 & 79.32 & 79.35 \\
500 & 210.7 & 210.7 & 58.37 & 58.23 & 78.62 & 78.70 \\
600 & 205.8 & 205.9 & 58.72 & 58.44 & 77.91 & 77.94 \\
700 & 200.9 & 201.2 & 59.07 & 58.66 & 77.20 & 77.18 \\
800 & 196.0 & 196.6 & 59.42 & 58.81 & 76.50 & 76.46 \\
900 & 191.1 & 192.0 & 59.77 & 58.98 & 75.79 & 75.72 \\
1000 & 186.2 & 187.2 & 60.12 & 58.98 & 75.09 & 74.92 \\
1100 & 181.3 & 182.7 & 60.47 & 58.96 & 74.38 & 74.17 \\
1200 & 176.5 & 178.1 & 60.82 & 58.99 & 73.67 & 73.48
\end{tabular}

TABLE VIII

$\mathrm{MgSiO}_{4}$ : Calculated values of elastic constants (in GPa) at different temperatures (top) along with the experimental data [2] (bottom) obtained under adiabatic condition. The first value of $C_{i j}$ comes from Eq. (15), the second one from [2].

\begin{tabular}{c|c|c|c|c|c|c|c|c|c}
\hline \hline$T[\mathrm{~K}]$ & $C_{11}$ & $C_{22}$ & $C_{33}$ & $C_{44}$ & $C_{55}$ & $C_{66}$ & $C_{23}$ & $C_{31}$ & $C_{12}$ \\
\hline 300 & 330.0 & 200.0 & 236.0 & 67.2 & 81.5 & 81.2 & 71.2 & 68.0 & 66.2 \\
& 330.0 & 200.0 & 236.0 & 67.2 & 81.5 & 81.2 & 71.2 & 68.0 & 66.2 \\
\hline \multirow{2}{*}{500} & 322.7 & 194.5 & 230.2 & 64.5 & 78.6 & 77.9 & 70.0 & 66.1 & 64.0 \\
& 322.4 & 194.2 & 230.1 & 64.4 & 78.7 & 78.0 & 71.1 & 66.1 & 64.0 \\
\hline 700 & 315.4 & 189.0 & 224.4 & 61.7 & 75.6 & 74.6 & 68.7 & 64.2 & 61.9 \\
& 314.5 & 188.0 & 223.6 & 61.6 & 75.8 & 74.6 & 69.7 & 64.3 & 61.8 \\
\hline \multirow{2}{*}{900} & 308.1 & 183.5 & 218.7 & 59.0 & 72.7 & 71.3 & 67.5 & 62.3 & 59.7 \\
& 306.3 & 181.5 & 216.9 & 58.8 & 72.8 & 71.3 & 68.3 & 62.5 & 59.4 \\
\hline \multirow{2}{*}{1100} & 300.9 & 177.9 & 212.9 & 56.2 & 69.8 & 68.0 & 66.2 & 60.5 & 57.6 \\
& 297.4 & 175.1 & 209.8 & 56.1 & 69.9 & 67.9 & 67.2 & 60.5 & 57.3 \\
\hline \multirow{2}{*}{1300} & 293.6 & 172.4 & 207.1 & 53.5 & 66.9 & 64.7 & 65.0 & 58.6 & 55.4 \\
& 288.3 & 168.7 & 202.7 & 53.3 & 66.9 & 64.6 & 66.0 & 58.5 & 55.3 \\
\hline \multirow{2}{*}{1500} & 286.3 & 166.9 & 201.4 & 50.8 & 64.0 & 61.4 & 63.8 & 56.7 & 53.3 \\
& 279.1 & 162.2 & 195.5 & 50.6 & 64.0 & 61.4 & 64.6 & 56.7 & 53.2 \\
\hline \multirow{2}{*}{1700} & 279.0 & 161.5 & 195.6 & 48.1 & 61.0 & 58.3 & 62.5 & 54.8 & 51.1 \\
& 269.8 & 155.6 & 188.2 & 48.0 & 61.0 & 58.4 & 63.3 & 54.9 & 51.0
\end{tabular}


temperature and thus produces a large change in $C_{11}$. On the other hand, the constants $C_{12}$ and $C_{44}$ are related to the elasticity in shape which is shear constant. A transverse strain or shearing causes a change in shape without a change in volume. Thus $C_{12}$ and $C_{44}$ are less sensitive of temperature as compared with $C_{11}$.

Equation (15) has also been extended to calculate the SOEC of more complicated minerals, viz. $\mathrm{Mg}_{2} \mathrm{SiO}_{4}$. For $\mathrm{Mg}_{2} \mathrm{SiO}_{4}$, there are nine SOEC, the calculated values of SOEC are also found to be in very good agreement with the experimental data [2]. The maximum deviation found for $C_{33}$ at highest temperature $(1700 \mathrm{~K})$ is $3.93 \%$.

To summarize, we have thus presented a simple and straightforward method to study the elastic properties of solids under the varying conditions of temperatures. The results obtained are encouraging and demonstrate that the present method is reasonable. Due to the simplicity of the method, it can be extended to more complex solids like minerals at higher temperature.

\section{Acknowledgments}

This work was financially supported by Key Laboratory of Opto-electronic Information Acquisition and Manipulation of Ministry of Education of China (No. 9603).

\section{References}

[1] O.L. Anderson, Equation of State of Solids for Geophysics and Ceramic Science, Oxford University Press, Oxford 1995.

[2] O.L. Anderson, D.G. Isaak, in: Mineral Physics and Crystallography: A Handbook of Physical Constants, Ed. T.J. Ahrens, WA: Am. Geophys. Union, Washington (DC) 1995, p. 64.

[3] D.G. Isaak, I. Ohno, Phys. Chem. Minerals 30, 430 (2003).

[4] S.V. Sinogeikin, D.L. Lakshtanov, J.D. Nicholas, J.M. Jackson, J.D. Bass, J. Europ. Ceram. Soc. 25, 1313 (2005).

[5] U.C. Shrivastava, Phys. Rev. B 21, 2602 (1980).

[6] R.J. Wolf, K.A. Mansour, M.W. Lee, J.R. Ray, Phys. Rev. B 46, 8027 (1992).

[7] X.F. Li, X.R. Chen, C.M. Meng, G.F. Ji, Solid State Commun. 139, 197 (2006).

[8] J.J. Zhao, J.M. Winey, Y.M. Gupta, Phys. Rev. B 75, 094105 (2007).

[9] J.L. Tallon, J. Phys. Chem. Solids 41, 837 (1980).

[10] O.L. Anderson, D.G. Isaak, J. Phys. Chem. Solids 54, 221 (1993).

[11] M. Kumar, Physica B 205, 175 (1995).

[12] J. Shanker, M. Kumar, Phys. Status Solidi B 179, 351 (1993).

[13] R. Grover, I.C. Getting, G.C. Kennedy, Phys. Rev. B 7, 567 (1973).

[14] M. Kumar, S.S. Bedi, High Temperatures-High Pressures 27/28, 595 (1995/1996).

[15] C. Wong, D.E. Schuele, J. Phys. Chem. Solids 29, 1309 (1968).

[16] C.R.A. Catlow, J.D. Comins, F.A. Germano, R.T. Harley, W. Hayes, J. Phys. C, Solid State Phys. 11, 3197 (1978). 